\title{
Asociación de infección por Chlamydia trachomatis con el diagnóstico de cervicitis aguda
} Association of Chlamydia trachomatis with the Diagnosis
of Acute Cervicitis

\section{Associação da infecção de Chlamydia trachomatis com o diagnóstico de cervicite aguda}

Juan Manuel Jaimes-Bravo, MD*

\section{Resumen}

Introducción: la infección por Chlamydia trachomatis es la enfermedad de transmisión sexual más frecuente en el mundo y causa una importante morbilidad en las mujeres. Actualmente existen pocos estudios sobre su prevalencia en nuestra población, por lo que se hace pertinente la implementación de un estudio piloto que aporte información sobre su frecuencia en mujeres asintomáticas y con cervicitis aguda. Objetivo: determinar la asociación existente entra la infección por Chlamydia trachomatis y la presencia de cervicitis aguda en la población consultante a los servicios de urgencias y consulta externa del Hospital Universitario de Santander (HUS). Metodología: estudio de casos y controles en el cual se incluyeron 284 pacientes mayores de edad, no embarazadas y que no estuvieran menstruando, atendidas en el HUS. Se tomaron como casos las pacientes con diagnóstico de cervicitis aguda y controles aquellas sin el mismo. En los dos grupos se realizó un frotis endocervical al cual se aplicó un método de diagnóstico rápido para infección por Chlamydia trachomatis, Bioline Chlamydia SD®. Resultados: el promedio de edad fue de 32.08 y 28.82 para casos y controles, con una diferencia promedio de 3.27 , $p=0.001$. También hubo diferencias al evaluar estado civil; $82.40 \%$ de los controles tiene un vínculo marital frente al
$71.83 \%$ de los casos, $p=0.014$; ocupación, más estudiantes en el grupo de cervicitis que en los casos, $p=0.020 ; y$ consumo de alcohol, $21.13 \%$ de pacientes con cervicitis, contra $11.27 \%$ de los controles, $p=0.024$. La prevalencia de infección por Chlamydia en los controles fue de $7.04 \%$ y $11.97 \%$ en los casos, $p=0.157$. Al buscar una asociación entre cervicitis aguda y la infección por Chlamydia esta no se encuentra, OR = 1.79 (IC 95\% $0.74-4.55)$. Esta falta se asociación se mantiene luego de ajustar los potenciales factores confusores (OR = 1.71, IC 95\% 0.72 - 4.04). En el análisis multivariado solo el consumo de alcohol persiste asociado con la presencia de cervicitis, OR $=4.442$ (IC 95\% 1.362 - 14.215). Conclusiones: no se encontró asociación entre la infección por Chlamydia trachomatis y el diagnóstico de cervicitis aguda. Se encontró una prevalencia similar en los dos grupos, además no se encontraron diferencias sociodemográficas. El único factor de riesgo con una asociación fuerte para colonización por la bacteria y cervicitis aguda fue la historia de consumo de alcohol. [Jaimes-Bravo JM. Asociación de infección por Chlamydia trachomatis con el diagnóstico de cervicitis aguda. MedUNAB 2015; 18 (2): 116-124]

Palabras clave: Cervicitis uterina; enfermedades del cuello del útero; Chlamydia trachomatis; Infecciones del Sistema Genital; Infecciones por Chlamydia.

* Médico General, Residente de Ginecología y Obstetricia, Universidad Industrial de Santander.

Correspondencia: Juan Manuel Jaimes Bravo. Correo electrónico: jmjaimesb@gmail.com 


\begin{abstract}
Introduction: Chlamydia trachomatis infection is the most frequent sexually transmitted disease in the world causing heightened morbidity in women. Currently there are few studies about its prevalence in our population; therefore, the implementation of a pilot study about its frequency in women with and without acute cervicitis is necessary. Objective: Determine the association between Chlamydia trachomatis infection and the diagnosis of acute cervicitis in the consulting women in both the outpatient and inpatient services of the Hospital Universitario de Santander (HUS). Method: Case control study including 284 patients of adult age, non-pregnant and without menses who consulted HUS. Cases with acute cervicitis diagnosis and routine control ones were considered for the purpose of this study. Endocervical samples and a quick diagnostic method for Chlamydia trachomatis, Bioline Chlamydia SD were applied. Results: Mean age was 32.08 and 28.82 years for cases and controls, mean difference was $3.27, p=0.001$. There were also differences in the evaluation of marital status; $82.40 \%$ controls have a marital relationship versus $71.83 \%$ cases, $p=0.014$; occupation, more students in the cervicitis group, $p=0.020$; and alcohol consumption, $21.13 \%$ in cervicitis patients versus $11.27 \%$ in the control group, $p=0.024$. Chlamydia prevalence was $7.04 \%$ in controls and $11.97 \%$ in cases, $p=0.157$. There was no association between acute cervicitis and Chlamydia infection, OR $=1.79$ (IC 95\% $0.74-4.55)$. This lack of association persists after adjusting for confusing factors (OR $=1.71$, IC 95\% $0.72-4.04$ ). In the multivariate analysis only alcohol consumption is associated with acute cervicitis, OR = 4.442 (IC 95\% $1.362-14.215)$. Conclusions: There was no association between Chlamydia trachomatis infection and acute cervicitis. Prevalence was similar for both groups; there were no socio-demographic differences. Only alcohol consumption was associated with bacterial colonization and acute cervicitis. [Jaimes-Bravo JM. Association of Chlamydia trachomatis with the Diagnosis of Acute Cervicitis. MedUNAB 2015; 18 (2): 116-124]
\end{abstract}

Keywords: Uterine Cervicitis; Uterine Cervical Diseases; Chlamydia trachomatis; Reproductive Tract Infections; Chlamydia Infections.

\section{Resumo}

Introdução: A infecção por Chlamydia trachomatis é a doença sexualmente transmissível mais comum no mundo, causando significativa morbidade nas mulheres. Atualmente, existem poucos estudos sobre sua prevalência nossa população, por isso torna-se relevante a implementação de um estudo piloto para fornecer informações sobre a sua frequência em mulheres assintomáticas com cervicite aguda. Objetivo: Determinar a associação entre a infecção por Chlamydia trachomatis e a presença da cervicite aguda nas pessoas que frecuentam e consultam no pronto socorro e o ambulatorio do Hospital Universitário de Santander (HUS). Metodologia: No estudo dos casos e nos controles de 284 pacientes foram incluídas mulheres idosas, não grávidas e as que não estavam no periodo menstrual atendidas pelo HUS. Para o estudo dos casos foram selecionadas aquelas com o diagnóstico de cervicite aguda e para o control as que não tinham a doença. Em ambos grupos é realizado um esfregaço endocervical para ao qual é aplicado um método de diagnóstico rápido para Chlamydia trachomatis, Chlamydia SD ${ }^{\circledR}$ Bioline. Resultados: A idade média foi de 32.08 e 28.82 para casos e controles, com uma diferença média de 3.27, $p=0.001$. Houve também diferenças na avaliação de estado civil; $82.40 \%$ dos controles têm um vínculo conjugal contra $71.83 \%$ dos casos, $p=0.014$; ocupação, mais alunos no grupo que, em casos cervicite, $p=0.020$; e consumo de álcool, $21.13 \%$ dos pacientes com cervicite, contra $11.27 \%$ dos controlos, $p=0.024$. A prevalência da infecção por Chlamydia nos controlos era $7.04 \%$ e $11.97 \%$ nos casos, $p=$ 0.157 . O estudo revelou que não ha uma associação entre cervicite aguda e infecção por Chlamydia, OR = 1.79 (IC $95 \%$ 0.74-4.55). Esta falta de associação permanece após o ajuste dos potenciais fatores de confusão $(O R=1.71, \mathrm{Cl}$ $0.7295 \%$ - 4.04). Na análise multivariada só o álcool permanece associado com a presença de cervicite, $\mathrm{OR}=$ (IC 95\% 1.362-14.215) 4.442. Conclusões: Não houve associação entre infecção por Chlamydia trachomatis e o diagnóstico de cervicite aguda. Foi encontrada uma prevalência semelhante nos dois grupos e não encontraram diferenças sociodemográficas. O único fator de risco com uma forte associação à colonização por bactérias e cervicite aguda foi a história de consumo de álcool. [Jaimes-Bravo JM. Associação da infecção de Chlamydia trachomatis com o diagnóstico de cervicite aguda. MedUNAB 2015; 18 (2): 116-124]

Palavras-chave: Cervicite Uterina; Doenças do Colo do Útero; Chlamydia trachomatis; Infecções do Sistema Genital; Infecções por Chlamydia.

\section{Introducción}

La infección por Chlamydia trachomatis es la enfermedad de transmisión sexual más frecuente en el mundo (1) que causa una importante morbilidad en las mujeres, incluyendo infertilidad, enfermedad pélvica inflamatoria, embarazos ectópicos y dolor pélvico crónico. (2) En los Estados Unidos la Chlamydia es responsable de aproximadamente cuatro millones de infecciones nuevas cada año. En Europa, esta infección ha alcanzado proporciones epidémicas, estimándose que, en algunos países, un tercio de la población puede tener una o más infecciones a lo largo de su vida (2). Un estudio realizado en México informó una prevalencia de $11.40 \%$ de anticuerpos IgG y de $4.40 \%$ de anticuerpos IgA anticlamidia entre 585 mujeres en edad reproductiva (3). En Brasil, un estudio que incluyó 627 hombres jóvenes asintomáticos reclutados para el servicio militar, mostró una prevalencia del 5\% (IC 95\% 3.7-7.3) de infección por C. trachomatis (4), y en el mismo país se encontró una prevalencia global de $8.90 \%$ (IC 95\% 6.50-11.90) entre 464 mujeres de 15 a 19 años de edad usando reacción en cadena de la ligasa para la detección de clamidia (5). García y colaboradores informaron una 
prevalencia de $6.80 \%$ de infección por Chlamydia detectada por PCR, entre 754 participantes en un estudio en Perú (6). Otro estudio realizado en Argentina con 279 mujeres mostró que la prevalencia de DNA para Chlamydia trachomatis aumentó desde $11 \%$ en mujeres con citología normal hasta $47.00 \%$ en mujeres con lesiones escamosas intraepiteliales de alto grado (7), mientras que en Bogotá (Colombia), Molano y colaboradores informaron una prevalencia global del $5.00 \%$ entre 1,829 mujeres sexualmente activas de bajos ingresos económicos, sin diferencias significativas entre quienes tenían citología normal y quienes tenían citología anormal (8).

La Chlamydia trachomatis es una bacteria intracelular obligada, gram negativa que pertenece al género Chlamydia familia Chlamydiaceae, orden Chlamydiales. La mayoría de las infecciones por Chlamydia son asintomáticas por lo que se dificulta hacer el diagnóstico temprano para evitar secuelas a largo plazo (9-11).

La cervicitis aguda se define como la inflamación aguda del cuello uterino. Esta inflamación afecta principalmente a las células columnares del endocérvix, pero también puede afectar las células escamosas del exocérvix. Normalmente es secundaria a una infección (Chlamydia, Neisseria, etc.); aunque no se puede determinar una infección específica en la mayoría de los casos. La cervicitis crónica normalmente no es de origen infeccioso y se hace este diagnóstico a partir del cuarto mes con sintomatología(12-15).

Cuando una causa infecciosa es detectada, se identifica la presencia de Chlamydia trachomatis o Neisseria gonorrhoeae en la mayoría de los casos. La cervicitis por Chlamydia ocurre más frecuentemente que la producida por gonococo $(2,11,16,17)$. Estas dos afectan principalmente a las células del endocérvix. También hay casos asociados con infección por el virus del herpes simple (VHS) y con Trichomona vaginalis, pero estas afectan principalmente a las células del exocérvix $(12,18)$.

Actualmente existen pocos estudios sobre la prevalencia de esta infección en nuestra población y la mayoría de los tratamientos instaurados son realizados de forma empírica, por lo que se hace pertinente la implementación de un estudio piloto que nos aporte información sobre su frecuencia en mujeres asintomáticas y con cervicitis aguda (2).
Determinar la asociación existente entre la infección por Chlamydia trachomatis y la presencia de cervicitis aguda en la población consultante a los servicios de urgencias y consulta externa del Hospital Universitario de Santander.

\section{Materiales y métodos}

Se realizó un estudio de casos y controles en el cual se incluyeron de forma consecutiva mujeres mayores de edad, no embarazadas consultantes a los servicios de consulta externa y urgencias del Hospital Universitario de Santander, quienes consultaron voluntariamente y aceptaron participar en el estudio mediante la firma del consentimiento informado. El protocolo siguió la normatividad local e internacional, respecto a los derechos de los pacientes y fue aprobado por el Comité de Ética en Investigación Científica de la Universidad Industrial de Santander y por el comité de Investigaciones del Hospital Universitario de Santander. Para el cálculo del tamaño de la muestra se utilizó una tabla de poder en la cual se tuvo en cuenta la prevalencia esperada de infección por Chlamydia en pacientes con y sin cervicitis, con un error alfa estimado del $5.00 \%$ y un error beta estimado del $20.00 \%$, para una proporción de 1:1 (ver Tabla 1). Teniendo en cuenta que se calcula una prevalencia en pacientes asintomáticas y con cervicitis aguda del $5.00 \%$ y $15.00 \%$ respectivamente; estimamos un tamaño de 142 pacientes para cada grupo del estudio, un control para cada caso.

El diagnóstico de cervicitis aguda es eminentemente clínico y se realizó de acuerdo con los siguientes criterios: (1) exudado cervical purulento o mucupurulento y (2) sangrado cervical (friabilidad) inducido fácilmente al tocar el área con un hisopo (13). La cervicitis crónica normalmente no es de origen infeccioso y se hace este diagnóstico a partir del cuarto mes con sintomatología con un tratamiento adecuado (13-15), por lo que este fue el factor determinante para excluir a las pacientes que no cumplieran este criterio.

Para la toma de la muestra se siguieron los siguientes pasos, previa realización de especuloscopia y se hiciera o no el diagnóstico de cervicitis aguda, momento en el que se asignará la paciente al grupo de casos o de controles según sea el caso:

Tabla 1. Tabla de poder para el cálculo del tamaño de muestra.

\begin{tabular}{cccccc}
\hline \multirow{2}{*}{$\begin{array}{c}\text { Prevalencia } \\
\text { en controles }\end{array}$} & \multicolumn{5}{c}{ Prevalencia en cervicitis } \\
\cline { 2 - 6 } & $5 \%$ & $10 \%$ & $15 \%$ & $20 \%$ & $25 \%$ \\
\hline $2 \%$ & 590 & 139 & 73 & 48 & 35 \\
$3 \%$ & 1507 & 195 & 90 & 56 & 40 \\
$4 \%$ & 6747 & 284 & 112 & 65 & 45 \\
$5 \%$ & - & 436 & 142 & 77 & 51 \\
\hline
\end{tabular}


Debe tomarse un primer hisopo para quitar el exceso de moco que se encuentre en el exocérvix. Después de limpiar el exocérvix, el segundo hisopo deberá introducirse en el canal endocervical, pasando por la unión escamo-columnar hasta cuando la mayor parte de la punta no sea visible. Girar firmemente el hisopo durante 2-3 segundos, dando dos vueltas completas. El hisopo debe retirarse sin contaminación de células exocervicales o vaginales.

(4) La muestra puede analizarse inmediatamente o devolverse al tubo suministrado para su almacenamiento o transporte. No colocar el espécimen en ningún dispositivo de transporte que contenga un medio que interfiera con el ensayo.

0 Las muestras se pueden almacenar durante seis horas a temperatura de $15-27^{\circ} \mathrm{C}, \mathrm{o} 72$ horas en refrigeración de $2-8^{\circ} \mathrm{C}$. Se recomienda que las muestras se procesen tan pronto como sea posible después de su recolección.

$\checkmark$ La prueba diagnóstica aplicada es Bioline Chlamydia $S D{ }^{\circledR}$ del laboratorio Standard Diagnostics Inc. Esta es una prueba de detección de antígenos por inmunocromatografía(DFA y EIA).

( Para su aplicación las muestras fueron llevadas y procesadas en el Laboratorio Central de Investigaciones en la facultad de salud de la Universidad Industrial de Santander.

Como criterios de inclusión se tomaron los siguientes:

Paciente sexualmente activa, mayor de edad, no embarazada, quien no se encuentre menstruando y que sea tratada en los servicios de urgencias o consulta externa del Hospital Universitario de Santander.
No tener historia de inmunosupresión o enfermedad grave y no haber recibido tratamiento para dicha enfermedad en el último mes.

( No haber recibido tratamiento antibiótico para otra patología que pueda ejercer algún efecto sobre la Chlamydia durante el último mes.

Los siguientes fueron los criterios de exclusión:

Muestra insatisfactoria para el análisis.

ㄱesultados del análisis no concluyentes.

El análisis estadístico de los resultados se llevó a cabo con el programa Stata 12.00 . Se realizó comparación en cuanto a las variables explicatorias, aceptando como nivel de significancia $\alpha<0.05$ mediante las pruebas correspondientes al tipo de variable y su naturaleza Gaussiana o no (v. gr. T de Student, Chi cuadrado, prueba de Wilcoxon). Razón de prevalencia con su IC 95\%.

\section{Resultados}

En total se reclutaron 284 pacientes: 142 en el grupo de casos y 142 en el grupo de controles. No se excluyó a ninguna paciente del estudio, puesto que quienes no cumplían con los criterios de inclusión no fueron invitadas a participar y, en este sentido, ninguna paciente seleccionada cumplió con los criterios de exclusión.

Características sociodemográficas:

Tabla 2. Características sociodemográficas de las pacientes.

\begin{tabular}{llll}
\hline Característica estudiada & Pacientes sanas & $\begin{array}{c}\text { Pacientes con } \\
\text { cervicitis }\end{array}$ & Valor de $\mathbf{p}$ \\
\hline Edad (promedio en años) & 32.08 & 28.82 & 0.001 \\
\hline $\begin{array}{l}\text { Procedencia } \\
\text { Área metropolitana de Bucaramanga }\end{array}$ & $115(80.99 \%)$ & $122(85.92 \%)$ & 0.446 \\
Otro municipio de Santander & $25(17.61 \%)$ & $15(10.56 \%)$ & \\
Fuera de Santander & $2(1.41 \%)$ & $5(3.52 \%)$ & \\
\hline Nivel educativo & & & 0.196 \\
Primaria & $55(38.73 \%)$ & $49(34.51 \%)$ & \\
Secundaria & $79(55.63 \%)$ & $82(57.75 \%)$ & \\
Técnica & $5(3.52 \%)$ & $2(1.41 \%)$ & \\
Universitaria & $3(2.11 \%)$ & $9(6.34 \%)$ & \\
\hline Ocupación & & & 0.020 \\
Hogar & $106(74.65 \%)$ & $90(63.38 \%)$ & \\
Estudiante & $2(1.41 \%)$ & $15(10.56 \%)$ & \\
Desempleada & $4(2.82 \%)$ & $0(0.00 \%)$ & \\
Empleada formal & $16(11.26 \%)$ & $25(17.50 \%)$ & \\
Empleada informal & $14(9.86 \%)$ & $12(8.56 \%)$ & \\
\hline
\end{tabular}




\begin{tabular}{llll}
\hline Característica estudiada & Pacientes sanas & $\begin{array}{c}\text { Pacientes con } \\
\text { cervicitis }\end{array}$ & Valor de p \\
\hline Estado civil & $19(13.38 \%)$ & $38(26.76 \%)$ & 0.014 \\
Soltera & $61(42.96 \%)$ & $48(33.80 \%)$ & \\
Casada & $56(39.44 \%)$ & $54(38.03 \%)$ & \\
Unión libre & $0(0.00 \%)$ & $1(0.70 \%)$ & \\
Viuda & $6(4.23 \%)$ & $1(0.70 \%)$ & \\
Separada & & & 0.22 \\
\hline Estrato socioeconómico & $10(7.04 \%)$ & $13(9.15 \%)$ & \\
Estrato 1 & $40(28.17 \%)$ & $24(16.90 \%)$ & \\
Estrato 2 & $45(31.69 \%)$ & $49(34.51 \%)$ & \\
Estrato 3 & $42(29.58 \%)$ & $52(36.62 \%)$ & \\
Estrato 4 & $5(3.52 \%)$ & $4(2.82 \%)$ & \\
Estrato 5 & & & \\
\hline
\end{tabular}

Edad: en el grupo de controles el promedio de edad es de 32.08 años con una desviación estándar de 8.93 años; mientras que en el grupo de casos es de 28.82 años con una desviación estándar de 7.61 años. $\mathrm{Al}$ aplicar la prueba de T de Student se encuentra una diferencia promedio de 3.27 (IC 95\% 1.33-5.21) años a favor de los controles (significando que estas son mayores), siendo esta significativamente estadística con una $\mathrm{p}=0.001$, ver tabla 2 .
Estado civil: en el grupo de pacientes control se encuentra que la mayoría, $117(82.40 \%)$ tiene un vínculo marital y una minoría, 25 (27.61\%) carece del mismo; en el grupo de pacientes con cervicitis se encuentra una distribución similar teniéndose el vínculo marital en $102(71.83 \%)$ y estando libres del mismo 40 (28.10\%). en este aspecto se encuentran diferencias estadísticamente significativas en los dos grupos con una $\mathrm{p}=0.014$, ver tabla 2 .

Tabla 3. Historia gestacional.

\begin{tabular}{|c|c|c|c|}
\hline & Pacientes sanas & Pacientes con cervicitis & Valor de $p$ \\
\hline Gravidez & & & 0.001 \\
\hline 0 & $6(4.23 \%)$ & $23(16.90 \%)$ & \\
\hline 1 & 32 (22.54\%) & 38 (26.76\%) & \\
\hline 2 & $41(28.87 \%)$ & 33 (23.24\%) & \\
\hline 3 & $33(23.24 \%)$ & 27 (19.01\%) & \\
\hline 4 & $18(12.68 \%)$ & 12 (8.45\%) & \\
\hline 5 o más & $12(8.45 \%)$ & $8(5.63 \%)$ & \\
\hline Partos & & & 0.025 \\
\hline 0 & 40 (28.17\%) & 58 (40.85\%) & \\
\hline 1 & $32(22.54 \%)$ & $30(21.13 \%)$ & \\
\hline 2 & $35(24.65 \%)$ & $28(19.72 \%)$ & \\
\hline 3 & $18(12.68 \%)$ & $18(12.68 \%)$ & \\
\hline 4 & 12 (8.45\%) & $3(2.11 \%)$ & \\
\hline 5 o más & $5(3.52 \%)$ & $5(3.52 \%)$ & \\
\hline Cesáreas & & & 0.071 \\
\hline 0 & 92 (64.79\%) & $106(74.65 \%)$ & \\
\hline 1 & $28(19.72 \%)$ & $19(13.38 \%)$ & \\
\hline 2 & $17(11.97 \%)$ & $14(9.86 \%)$ & \\
\hline 3 & $5(3.52 \%)$ & $3(2.11 \%)$ & \\
\hline Abortos & & & 0.256 \\
\hline 0 & 106 (74.65\%) & $114(80.28 \%)$ & \\
\hline 1 & $32(22.54 \%)$ & $23(16.20 \%)$ & \\
\hline 2 & $3(2.11 \%)$ & $3(2.11 \%)$ & \\
\hline 3 & $1(0.70 \%)$ & $1(0.70 \%)$ & \\
\hline 4 & $0(0.00 \%)$ & $1(0.70 \%)$ & \\
\hline Ectópicos & & & 0.310 \\
\hline 0 & $139(97.89 \%)$ & 136 (95.77\%) & \\
\hline 1 & $3(2.11 \%)$ & $6(4.23 \%)$ & \\
\hline
\end{tabular}


Ocupación: se encuentra que en el grupo de pacientes con cervicitis aguda la ocupación más frecuente es el hogar, $90(63.38 \%)$ casos, seguida por otras ocupaciones, 23 (16.20\%) casos, y estudiantes, $15(10.56 \%)$ casos, comerciante, $8(5.63 \%)$ casos y profesional, $6(4.23 \%)$ casos. En el grupo de controles la ocupación más frecuente es el hogar, $106(74.65 \%)$ casos, seguida por otras ocupaciones $19(13.38 \%)$ casos, comerciante, 8 $(5.63 \%)$ casos, profesional, $7(4.93 \%)$ casos, y estudiante, $2(1.41 \%)$ casos. Se encuentra una diferencia significativamente estadística entre los grupos, $p=0.020$, esta diferencia es más marcada en el subgrupo de estudiantes y mujeres dedicadas al hogar, ver tabla 2.

- Para nivel socioeconómico, régimen de afiliación a la seguridad social, escolaridad, lugar de residencia y procedencia no se encontraron diferencias estadísticamente significativas, ver tabla 2.

$\checkmark$ Número de embarazos: al clasificar las pacientes en aquellas sin historia obstétrica y las que tenían historia obstétrica se encuentra que en el grupo de controles hay $6(4.23 \%)$ nuligestantes y $136(95.77 \%)$ multigestanes; mientras que en el grupo de casos hay $24(16.90 \%)$ nuligestantes y $118(83.10 \%)$ multigestantes. Se encuentra una diferencia estadísticamente significativa entre ambos grupos, $\mathrm{p}=0.001$, habiendo más nuligestantes en los casos, ver tabla 3.

Número de partos: para simplificar el análisis se clasificaron entre nulíparas y multíparas, encontrándose en el grupo de casos $58(40.85 \%)$ nulíparas y 84
(59.15\%) multíparas; en el grupo de pacientes sanas hay $40(28.17 \%)$ nulíparas y 102 (71.83\%) multíparas. Acá se encuentra una diferencia significativa, $p=0.025$, para la nuliparidad, tabla 3 .

$\square$ No hubo diferencia estadísticamente significativa para el número de cesáreas, número de abortos y número de embarazos ectópicos, ver tabla 3.

Factores de riesgo para cervicitis:

No se encontraron diferencias estadísticamente significativas para la edad de inicio de relaciones sexuales, número de parejas sexuales, antecedente de enfermedades de transmisión sexual, antecedente de enfermedad pélvica inflamatoria, uso del preservativo ni consumo de sustancias psicoactivas, ver tabla 4 .

0 Consumo de alcohol: en el grupo de pacientes con cervicitis aguda, $30(21.13 \%)$ manifiestan consumo frecuente de alcohol y 112 (78.87\%) niegan consumir alcohol. . En el grupo de pacientes sanas, 16 (11.27\%) refieren consumo de alcohol, y $126(88.73 \%)$ niegan consumir esta sustancia. En este caso se encuentra una diferencia estadísticamente significativa entre los dos grupos $21.13 \%$ contra $11.27 \%$ con una $p=0.024$, ver tabla 4.

Al evaluar la positividad de la prueba en el grupo de controles se encuentra que esta es positiva en $10(7.04 \%)$ de las pacientes y negativa en $132(92.96 \%)$ de ellas. En el

Tabla 4. Factores de riesgo para enfermedades de transmisión sexual

\begin{tabular}{lccc}
\hline Factor & Pacientes sanas & Pacientes con cervicitis & Valor de $\mathbf{p}$ \\
\hline Edad inicio de vida sexual & 17.93 & 17.43 & 0.139 \\
\hline Número de parejas sexuales & & & 0.338 \\
1 & $52(36.62 \%)$ & $51(35.92 \%)$ & \\
$2-3$ & $65(45.77 \%)$ & $63(44.37 \%)$ & \\
$4-5$ & $20(14.08 \%)$ & $16(11.27 \%)$ & \\
6 o más & $5(3.52 \%)$ & $12(8.45 \%)$ & 0.834 \\
\hline Antecedente de ETS & $12(8.45 \%)$ & $13(9.15 \%)$ & \\
$\mathrm{Si}$ & $130(91.55 \%)$ & $129(90.85 \%)$ & \\
No & & & \\
\hline Antecedente de EPI & $9(6.34 \%)$ & $8(5.63 \%)$ & 0.802 \\
$\mathrm{Si}$ & $133(93.66 \%)$ & $134(94.37 \%)$ & \\
No & & & \\
\hline Uso de preservativo & $23(16.20 \%)$ & $29(20.42 \%)$ & 0.024 \\
$\mathrm{Si}$ & $119(83.80 \%)$ & $113(79.58 \%)$ & \\
No & $16(11.27 \%)$ & $30(21.13 \%)$ & 0.063 \\
\hline Consumo de alcohol & $126(88.73 \%)$ & $112(78.87 \%)$ & \\
$\mathrm{Si}$ & & & \\
No & $4(2.82 \%)$ & $11(7.75 \%)$ & \\
\hline Consumo de psicoactivos & $138(97.18 \%)$ & $131(92.25 \%)$ & \\
$\mathrm{Si}$ & &
\end{tabular}


Tabla 5. Resultado de la prueba diagnóstica para infección por Chlamydia trachomatis.

\begin{tabular}{lcc}
\hline Resultado & Pacientes sanas & Pacientes con cervicitis \\
\hline Positiva & $10(7.04 \%)$ & $17(11.97 \%)$ \\
\hline Negativa & $132(92.36 \%)$ & $125(88.03 \%)$ \\
\hline
\end{tabular}

grupo de pacientes con cervicitis aguda la prueba fue positiva en 17 (11.97\%) pacientes y negativa en 125 $(88.03 \%)$ pacientes. No se encontraron diferencias estadísticamente significativas entre los grupos, $\mathrm{p}=0.157$.

Al buscar una relación directa entre la presencia de cervicitis aguda y la infección por Chlamydia trachomatis el OR obtenido es de 1.79 (IC 95\% 0.74-4.55), lo cual indica que no hay una asociación directa entre estos dos eventos. Esta falta de asociación se mantiene luego de ajustar por los potenciales factores confusores $(\mathrm{OR}=1.71$, IC $95 \% 0.72$ 4.04).

Todos menos uno de los factores potencialmente confusores que se encontraron asociados en el análisis bivariado, dejan de estarlo en el modelo multivariado: edad $(\mathrm{OR}=1.007, \mathrm{IC} 95 \%$ 0.949 - 1.068), número de embarazos $(\mathrm{OR}=0.840, \mathrm{IC} 95 \% 0.583-1.212)$, número de cesáreas $(\mathrm{OR}=0.977, \mathrm{IC} 95 \% 0.577-1.654)$. El único factor que persiste asociado con la presencia de cervicitis es la historia de consumo de alcohol $(\mathrm{OR}=4.442$, IC 95\% $1.362-$ 14.215). El modelo logístico ajusta adecuadamente: $\mathrm{X} 2=$ $214.35,228$ grados de libertad, $\mathrm{p}=0.733$.

\section{Discusión}

Este estudio tuvo por objetivo determinar si la infección por Chlamydia trachomatis se encuentra asociada con el diagnóstico de cervicitis aguda utilizando la metodología de casos y controles. Al evaluar la positividad de infección por Chlamydia en el análisis inicial no se encuentran diferencias estadísticamente significativas entre las pacientes sanas y aquellas con diagnóstico de cervicitis aguda; al buscar una relación directa entre la infección por Chlamydia y la presencia de cervicitis aguda esta tampoco se encuentra. Una vez se realiza el ajuste con los potenciales factores confusores en el análisis multivariado, de aquellos que se encontraban asociados, el único que persiste con esta asociación es la historia de consumo de alcohol; lo cual no se corresponde con lo descrito en los estudios de Arraíz y Corsenac $(19,20,21)$, quienes encontraron que los principales factores de riesgo asociados con la presencia de infecciones de transmisión sexual fueron el pertenecer a un grupo de edad joven (18-25 años), ser soltera, tener un bajo nivel educativo y tener un nivel socioeconómico bajo.

De acuerdo con los resultados del estudio se encuentra una prevalencia total de infección por Chlamydia trachomatis del $10.50 \%$, esta es del $7.04 \%$ en el grupo de pacientes sanas y del $11.97 \%$ en el grupo de pacientes con cervicitis. Estos valores son similares a los descritos en la literatura, aunque esta prevalencia es menor en los estudios de Hilger y Corbeto $(16,22)$ quienes reportan una prevalencia total alrededor del $2.50 \%$ y una prevalencia alrededor del $7 \%$ en pacientes sintomáticas; sin embargo, estos resultados son similares a lo descrito en estudios locales por Arraiz y Angel $(2,20,21)$ quienes en estudios similares encuentran que en las pacientes asintomáticas la prevalencia es de $7.40 \%$, $5.80 \%$ y $3.40 \%$ respectivamente; mientras que en las pacientes sintomáticas es de $13.70 \%, 9.90 \%$ y $7.80 \%$, respectivamente; los resultados de estos estudios tampoco encuentran diferencias estadísticamente significativas entre los grupos. Estos resultados similares en poblaciones latinoamericanas con gran parecido a la que se tomó en el presente estudio podrían obedecer a un comportamiento diferente en cuanto a la distribución de enfermedades de transmisión sexual en nuestra población, diferentes comportamientos de nuestra población con respecto a otras latitudes o a un perfil epidemiológico diferente por parte de los microorganismos implicados.

Se encontraron diferencias significativas al evaluar el estado civil y la ocupación; a pesar de que en los dos grupos fue más frecuente la presencia de un vínculo marital formal, la proporción entre tenerlo y no tenerlo fue mayor en el grupo control que en el de las pacientes con cervicitis aguda, siendo esta diferencia estadísticamente significativa; esto se podría relacionar de forma indirecta con el estudio de Corbeto (22) quien encuentra como principales factores de riesgo para infección por Chlamydia la presencia de una nueva pareja sexual en los últimos tres meses, el consumo de tabaco, y un nivel socioeconómico bajo. En cuanto a la ocupación, también se encontró una diferencia significativa, siendo la presencia de cervicitis aguda más frecuente entre las estudiantes al compararlas con los otros subgrupos evaluados. Esta asociación concuerda con lo descrito en la literatura, donde la presencia de cervicitis aguda e infecciones de transmisión sexual es mucho más frecuente en mujeres jóvenes y sin una relación estable (11, $16,19,23)$.

Al evaluar el número de embarazos se encontró que el diagnóstico de cervicitis aguda fue más frecuente en nuligestantes que en multigestantes, siendo significativa esta diferencia; sin embargo, al evaluar por el número de partos, cesáreas y abortos solo se encontraron diferencias significativas en el número de partos, donde la cervicitis 
aguda fue más frecuente en las nulíparas. Llama la atención que esta diferencia no se mantuvo al evaluar el número de cesáreas, abortos y embarazos ectópicos. Estos hallazgos son similares a lo descrito por Sing, Schmidt, Giakomelou y Ujević quienes reportan una mayor asociación entre la positividad para infección por Chlamydia trachomatis en pacientes con cervicitis mucupurulenta y el hecho de ser nulíparas (prevalencia del 59.80\%) y tener una pareja sexual con menos de seis meses de antigüedad, (prevalencia del $35 \%)(24-26)$.

Al analizar los factores de riesgo conocidos para cervicitis aguda (25) se encuentra que la edad de inicio de relaciones sexuales fue similar en los dos grupos. No hubo diferencias en número de parejas sexuales, antecedente de ETS, antecedente de EPI, uso de preservativo o consumo de sustancias psicoactivas. Se encontró una diferencia significativa en el consumo de alcohol, siendo este más frecuente en las pacientes con cervicitis aguda; sin embargo, en todos estos hallazgos en contra de frente a lo descrito por Land, Ross, Casey y van Liere quienes reportan que la presencia de cualquiera de estos antecedentes se relaciona con una mayor probabilidad de infección por Chlamydia trachomatis con un OR alrededor del 4.10 para el antecedente de ETS, 4.24 para consumo de sustancias psicoactivas, y de 2.28 para dos o más parejas sexuales.(23,27-29)

Entre las principales debilidades del estudio se encuentra el hecho de que el tamaño de la muestra no es lo suficientemente grande como para realizar generalizaciones de nivel poblacional; sin embargo, sí permite realizar aproximaciones iniciales a la situación actual de la población en la ciudad de Bucaramanga y su área metropolitana. Otra potencial debilidad del estudio podría encontrarse en la prueba diagnóstica utilizada, puesto que esta no corresponde al estándar de oro actual, aunque de acuerdo a la revisión de la literatura realizada la especificidad de la misma es similar, pero su sensibilidad es mucho menor (23).

Otra debilidad del estudio se relaciona con el hecho de que el diagnóstico de cervicitis aguda se realizó únicamente por clínica, lo cual corresponde a la definición actual del diagnóstico para cervicitis aguda; pero este diagnóstico fue hecho por distintas personas, razón por la que se podría esperar que pacientes con ectropión u otras alteraciones similares hubieran sido clasificadas como pacientes con cervicitis aguda, cuando en realidad eran sanas; este hecho podría alterar los resultados del estudio y por ende explicar las diferencias que se encontraron respecto a lo descrito en la literatura.

Como fortaleza se puede destacar el hecho de que la muestra recolectada a pesar de no tener un tamaño suficientemente grande, sí recoge pacientes de varias clases sociales y su distribución es bastante uniforme en cuanto a nivel de escolaridad, profesión, estado civil y ocupación; se puede afirmar entonces que estos resultados iniciales podrían ser extrapolados a la población general de esta región para así plantear estudios más grandes posteriormente.

\section{Conclusiones}

No se encontró una asociación entre la infección por Chlamydia trachomatis y el diagnóstico de cervicitis aguda en la población estudiada. Se halló una prevalencia similar en las pacientes con y sin cervicitis aguda; de otra parte, no se encontraron diferencias sociodemográficas significativas para ambos grupos. El único factor de riesgo que se encontró con una asociación significativa para colonización por la bacteria y cervicitis aguda fue la historia de consumo de alcohol.

No se encontraron diferencias sociodemográficas estadísticamente significativas entre las pacientes sanas colonizadas por Chlamydia trachomatis y las pacientes con diagnóstico de cervicitis aguda que tenían esta infección. Al comparar el grupo de pacientes sanas como un todo contra frente al grupo de pacientes con diagnóstico de cervicitis aguda, en cuanto a las características sociodemográficas de los mismos; no se encontraron diferencias significativas entre los mismos.

El diagnóstico de cervicitis aguda es más frecuente en mujeres sin un vínculo marital estable y en aquellas que estudian. Una posible causa de esta situación podría ser que en dichos grupos hay más tendencia a tener un mayor número de parejas sexuales y a consumir alcohol con mayor frecuencia.

A partir de los resultados del presente trabajo se puede establecer toda una nueva línea de investigación para el estudio de la prevalencia de las distintas enfermedades de transmisión sexual en la población local; al menos las más frecuentes según la literatura. Estudiar las características de los distintos grupos poblacionales donde se encuentran con más frecuencia; las complicaciones que estas generan (infertilidad, dolor pélvico crónico, embarazos ectópicos secundarios); los antimicrobianos a los que estas infecciones son susceptibles; patrones de comportamiento de la población local que ocasionan las diferencias encontradas con la literatura mundial, etcétera.

\section{Referencias}

1. Ujević B, Habek JC, Habek D. Prevalence of infection with Neisseria gonorrhoeae or Chlamydia trachomais in acute mucopurulent cervicitis. Arh Hig Rada Toksikol. 2009; 60(2): 197-203.

2. Sánchez RM, Ruíz Parra Al, Ostos Ortiz OL. Prevalencia de Chlamydia trachomatis detectada por reacción en cadena de la polimerasa en un grupo de mujeres jóvenes sintomáticas y asintomáticas en Bogotá, Colombia. Revista Colombiana de Obstetricia y Ginecología. 2006; 57(3): 171-81. 
3. Cravioto $\mathrm{M}$ del C, Matamoros O, Villalobos-Zapata $\mathrm{Y}$, Peña O, García-Lara E, Martínez M, et al. Prevalencia de anticuerpos anti-Chlamydia trachomatis y anti-Neisseria gonorrhoeae en poblaciones Mexicanas. Salud Pública Mex. 2003; 45 Supp 5: S681-9.

4. Fioravante FC, Costa Alves M de F, Guimaraes EM, Turchi MD, Freitas HA, Domingos LT. Prevalence of Chlamydia trachomatis in asymptomatic Brazilian military conscripts. Sex Transm Dis. 2005; 32: 165-9.

5. Miranda AE, Szwarcwald CL, Peres RL, Page-Shafer K. Prevalence and risk behaviors for chlamydial infection in a population-based study of female adolescents in Brazil. Sex Transm Dis. 2004; 31: 542-6.

6. Garcia PJ, Chavez S, Feringa B, Chiappe M, Li W, Jansen $\mathrm{KU}$, et al. Reproductive tract infections in rural women from the highlands, jungle, and coastal regions of Peru. Bull World Health Organ. 2004; 82: 483-92.

7. Golijow CD, Abba MC, Mouron SA, Laguens RM, Dulout FN, Smith JS. Chlamydia trachomatis and Human papillomavirus infections in cervical disease in Argentine women. Gynecol Oncol. 2005; 96: 181-6.

8. Molano M, Weiderpass E, Posso H, Morre SA, Ronderos M, Franceschi S, et al. HPV Study Group. Prevalence and determinants of Chlamydia trachomatis infections in women from Bogota, Colombia. Sex Transm Infect. 2003; 79: 474-8.

9. Ghosh M, Choudhuri S, Ray RG, Bhattacharya B. Association of Genital Chlamydia trachomatis Infection with Female Infertility, Study in a Tertiary Care Hospital in Eastern India. Open Microbiology Journal. 2015; (9): 110-6.

10. Matiluko A, Crystal A. Chlamydia in colposcopy clinics: to screen or not to screen? Journal of Obstetrics and Gynaecology. 2006; 26(5): 454-6.

11. Paavonen J. Chlamydia trachomatis infections of the female genital tract: state of the art. Annals of Medicine. 2012; 44: 18-28.

12. Santer M, Wyke S, Warner P. Women's experiences of Chlamydia screening. Qualitative interviews with women in primary care. The European Journal of General Practice. 2003; 9(2): 56-61.

13. Marrazo J. Acute cervicitis. Up to Date. 2012.

14. The overall agreement of proposed definitions of mucopurulent cervicitis in women at high risk of Chlamydia infection. Acta Dermato-venereologica. 2010; 90: 506-11.

15. Wright TC, Ronnett BM, Ferenczy A. Benign Diseases of the Cervix. In: Kurman RJ, Ronnett BM, Hedrick Ellenson L, editores. Blaustein's Pathology of the Female Genital Tract. Sixth edition. New York: Springer; 2010. p. 156-93.

16. Hilger TM, Smith EM, Ault K. Predictors of Chlamydia trachomatis infection among women attending rural Midwest family planning clinics. Infectious Diseases in Obstetrics and Gynecology. 2001; 9:3-8.

17. Ángel Müller E, González MP, Núñez L, Pacheco J, Tolosa JE, Díaz LA, et al. Frecuencia de infecciones del tracto genital femeninno en mujeres sintomáticas y uso de pruebas rápidas para su diagnóstico en dos poblaciones de Bogotá (Colombia) 2008. Estudio piloto. Revista Colombiana de Obstetricia y Ginecología. 2010; 61(3): 220-30.

18. Songur Dagli S, Demir T. Comparison of cervico-vaginal colonization among sexually active women by intrauterine device use. Journal of Infectious Diseases in Developing Countries. 2015; 9(9): 930-5

19. Corsenac $P$, Noël M, Rouchon B, Hoy D, Roth A. Prevalence and sociodemographic risk factors of Chlamydia, Gonorrhoea and Syphilis: a national multicentre STI survey in New Caledonia. BMJ Open. 2015; (5):1-9.

20. Arráiz N, Marcucci R, Colina S, Reyes F, Rondón N, Bermúdez V, et al. Infección por Chlamydia trachomatis en mujeres consultantes en Maracaibo, Venezuela. Revista de Salud Pública. 2008; 10(4): 615-24.

21. Arráiz R, Ginestre P, Perozo M, Castellano G, Urdaneta B, García G. Diagnóstico molecular y prevalencia de infecciones por Chlamydia trachomatis en pacientes sintomáticas y asintomáticas de una población del estado de Zulia, Venezuela. Revista Chilena de Infectología. 2007; 24(1):48-52.

22. Corbeto EL, Lugo R, Martró E, Falguera G, Ros $R$, Avecilla $A$, et al. Prevalencia de la infección por $C$. trachomatis y $N$. gonhorroeae y determinantes para su adquisición en jóvenes y adultos-jóvenes en Cataluña. Enfermedades Infecciosas y Microbiología Clínica. 2011; 29(2): 96-101.

23. Land, JA., Van Bergen, JE., Morré, SA., Postma M. Epidemiology of Chlamydia trachomatis infection in women and the cost-effectiveness of screening. Human Reproduction Update. 2010; 16: 189-204.

24. Sing D, Marrazzo JM. Screening and Management of Genital Chlamydial Infections. Infectious Diseases Clinics of North America. 2013; 27(4): 739-53.

25. Schmidt R, Rosetti Muniz R, Cola E, Stauffert D, Freitas Silveira M, Miranda AE. Maternal Chlamydia trachomatis Infections and Preterm Births in a University Hospital in Vitoria, Brazil. PLoS One. 2015; 10(10): 4-11.

26. Giakoumelou S, Wheelhouse N, Cuschieri K, Entrican G, Howie SEM, Horne AW. The role of infection in miscarriage. Human Reproduction Update. 2016; 22(1): 116-33.

27. Ross J, Judlin P, Jensen J. 2012 European guideline for the management of pelvic inflammatory disease. International Journal of Sexually Transmitted Diseases and AIDS. 2014; 25(1): 1-7.

28. Casey CG, Rutledge TF, Boyd MF, Starr TM. Sexually Transmitted Diseases Treatment Guidelines. Centers for Disease Control and Prevention. 2010; 59:1-116.

29. Van Liere G, van Rooijen MS, Hoebe CJ, Heijman T, de Vries HJ, Dukers-Muijrers NH. Prevalence of and Factors Associated with Rectal-Only Chlamydia and Gonorrhoea in Women and in Men Who Have Sex with Men. PLoS One. 2015; 10(10): 1-17. 